\title{
Association between osteoporosis and hepatitis B cirrhosis: a case-control study
}

\author{
Yijin Zhang ${ }^{{ }^{11}}$, Xuesong Gao ${ }^{{ }^{11}}$, Ting $\mathrm{Liu}^{2}$, Ping Gao ${ }^{1}$, Hongjie $\mathrm{Li}^{1}$, Nan Liu ${ }^{1}$, \\ Lili Gao', Gang Wan', Yaonan Zhang ${ }^{4}$, Xuefei Duan ${ }^{1}$
}

1. Department of General Medicine, Beijing Ditan Hospital, Capital Medical University, Beijing, China.

2. Clinical data and sample repository, Beijing Ditan Hospital, Capital Medical University, Beijing, China.

3. Department of Medical Record, Beijing Ditan Hospital, Capital Medical University, Beijing, China.

4. Department of Orthopedics, Beijing Hospital, National Center of Gerontology, Beijing, China.

qThese authors contributed equally to this work.

\begin{abstract}
Background and aims: Hepatitis B virus (HBV)-related cirrhosis is associated with decreased bone mineral density (BMD); however, the mechanism is yet unknown. To assess the incidence of osteoporosis in patients with HBV-associated cirrhosis and relevant mechanisms.

Methods: A total of 80 hospitalized patients with HBV-associated cirrhosis and 80 healthy controls were enrolled. The levels of serum osteocalcin, total procollagen type 1 amino-terminal propeptide, $\beta$-C-terminal telopeptide of type I collagen ( $\beta$-CTX), and 25-hydroxy vitamin D3 (25(OH)D3) was evaluated in the cirrhosis group.

Results: The BMDs of the lumbar spine $(\mathrm{P}<0.001)$ and hip joints $(\mathrm{P}=0.015)$ in the cirrhosis group were significantly lower than those in the controls. The incidence of osteoporosis in the cirrhosis group was significantly higher than that in the control group $(\mathrm{P}<0.001)$. Compared to the patients of the Child-Pugh grade A and $\mathrm{B}$, the BMD of lumbar spine and 25(OH)D3 was significantly decreased in patients of grade $\mathrm{C}$, while $\beta$-CTX was elevated. Patients in the cirrhosis group faced a higher risk of osteoporosis as compared to the controls $(\mathrm{P}<0.001)$.

Conclusions: Enhanced bone resorption accounted for increased risk of osteoporosis in severe cirrhosis. Thus, HBV-associated cirrhosis was a risk factor for osteoporosis.

Keywords: Liver cirrhosis; bone density; osteoporosis; osteopenia; hepatitis B, chronic.

DOI: https://dx.doi.org/10.4314/ahs.v20i4.13

Cite as: Zhang Y, Gao X, Lin T, Gao P, Li H, Liu N, et al. Association between osteoporosis and hepatitis B cirrhosis: a case-control study. Afri Health Sci. 2020;20(4):1610-6. https:// dx.doi.org/10.4314/abs.v20i4.13
\end{abstract}

\section{Introduction}

Approximately 248 million individuals are affected by chronic hepatitis $\mathrm{B}(\mathrm{CHB})$, globally. The number of patients with HBV-associated cirrhosis, hepatic decompensation, and deaths was 371,100 in $2015^{1}$. Hepatic osteodystrophy is a non-liver complication of chronic

\section{Corresponding authors: \\ Yaonan Zhang \\ Department of Orthopedics, Beijing Hospital, \\ National Center of Gerontology, Beijing, China \\ E-mail: zhangyn@vip.sina.com \\ And \\ Xuefei Duan \\ Department of General Medicine, \\ Beijing Ditan Hospital, Capital Medical University, \\ Beijing, China \\ Tel: +86-13311337433 \\ E-mail: duanxuefei@vip.sina.com}

liver disease, especially in patients with cirrhosis. Osteoporosis is the most common form of hepatic osteodystrophy and is associated with the increased risk of fragility fractures that lead to social and economic burdens ${ }^{2}$. Vertebral fractures are the most common types in patients with chronic liver disease ${ }^{3}$.

Recent studies mainly focused on the association between primary biliary cirrhosis or alcoholic cirrhosis and osteoporosis ${ }^{4-6}$. The pathogenesis of $\mathrm{CHB}$ patients with cirrhosis complicated with osteoporosis has not yet been well studied.

Thus, the present study aimed to evaluate the correlation between decreased bone mineral density (BMD) and bone markers to explore the related pathogenesis in $\mathrm{CHB}$ patients with cirrhosis.

\section{Subjects and methods Study design}

In this case-control study, $80 \mathrm{CHB}$ patients with cirrhosis hospitalized in the Department of General Medicine
African

1610

(C) 2020 Zhang Y et al. Licensee African Health Sciences. This is an Open Access article distributed under the terms of the Creative commons Attribution License (https://creativecommons.org/licenses/BY/4.0), which permits unrestricted use, distribution, and reproduction in any medium, provided the original work is properly cited. 
of Beijing Ditan Hospital of Capital Medical University between March 2015 and 2017 were selected as the subjects. Te inclusion criteria included patients with hepatitis B cirrhosis who were diagnosed by laboratory examination, histology, or imaging according to the guidelines of the American Association for the Study of Liver Diseases ${ }^{7}$. Also, the medical history of the patients including smoking history, age at menopause, history of drug abuse, family history of osteoporosis, eating habits, comorbidities, and history of antiviral therapy with HBV was collected. In addition, 80 healthy individualswere enrolled as controls from the Beijing Hospital Health Examination Center. The exclusion criteria for both groups were as follows: diseases affecting bone metabolism (for instance, parathyroid diseases, thyroid diseases, inherited disorders of connective tissue, sarcoidosis, or inflammatory bowel disease), patients withdual-energy X-ray absorptiometry(DXA) contraindications,patients taking estrogens or proton pump inhibitors, any medications that could affect the bone mass (for example, calcium, vitamin D supplements, corticosteroids), immunosuppressant therapy without organ transplant, history of malignant tumor, severe heart disease, chronic renal insufficiency, human immunodeficiency virus (HIV) co-infection, hepatitis $\mathrm{C}$ virus co-infection, autoimmune liver disease, primary biliary cirrhosis, and alcoholic liver disease.

This study was approved by the Institutional Review Board of the Beijing Ditan Hospital, Capital Medical University. Written informed consent was obtained from all participants (2015-041-01).

\section{BMD measurements and laboratory examination}

BMD $(\mathrm{g} / \mathrm{cm} 2)$ of the lumbar spine and the proximal hip (femoral neck, trochanter, and total hip) were measured by dual-energy X-ray absorptiometry (DXA) (QDR-4500A; Hologic, Waltham, MA, USA). Osteoporosis was defined as a $\mathrm{T}$ score below -2.5 standard deviations (SD), while osteopenia was defined as a $\mathrm{T}$ score between -1 and $-2.5 \mathrm{SD}^{8}$.

The levels of serum osteocalcin (OC), $\beta$-C-terminal telopeptides of type-I collagen ( $\beta$-CTX), procollagen type 1 amino-terminal propeptide (P1NP), and 25-hydroxyvitamin D3 (25(OH)D3) were measured using electrochemiluminescence immunoassay (Roche Diagnostics $\mathrm{GmbH}$, Mannheim, Germany). $\beta$-CTX is a degradation product of type 1 collagen and released into the circulation during bone resorption'. On the other hand, procollagen type 1 amino-terminal propeptide $(\mathrm{P} 1 \mathrm{NP})$ is a bone formation marker that is released into the circulation during bone formation". Osteocalcin is a polypeptide secreted primarily by osteoblasts into the bone matrix, and hence, is considered to be a marker of bone formation(10). 25(OH)D3 reflects the nutritional level of vitamin D.

Child-Pugh scores were used for the clinical staging of cirrhosis: A (5-6), B (7-9), and C (10-15), including five indicators: hepatic encephalopathy (phase), ascites, total bilirubin $(\mu \mathrm{mol} / \mathrm{L})$, albumin $(\mathrm{g} / \mathrm{L})$, and prothrombin time extension (s). The total bilirubin and albumin levels were tested using a Hitachi 7600 fully automatic biochemical analyzer (Wako Pure Chemical Industries Ltd, Tokyo, Japan). The prothrombin time was tested using an automatic blood coagulation instrument (CA6000, Sysmex Corporation, Japan).

\section{Statistical analysis}

Quantitative data were expressed as mean \pm SD. Qualitative data were reported as proportion (\%). The chisquare test was used for categorical variables. Student's t-test was utilized to compare data between two groups, and the Kruskal-Wallis test was used for comparisons across three groups with respect to continuous variables. $\mathrm{P}<0.05$ was considered statistically significant. Logistic regression analysis was used for the risk factors of osteoporosisanalyzed by thestepwise regression method, and the variables were selected using the backward method. All statistical tests were carried out by SPSS version 17 (SPSS Inc., Chicago, IL, USA).

\section{Results}

The clinical characteristics of the HBV cirrhotic patients and controls are summarized in Table 1. The cohort consisted of $44(55.0 \%)$ males and $36(45.0 \%)$ females in the HBV cirrhotic group and $40(50 \%)$ males and 40 $(50 \%)$ females in the control group. The mean age of the control group (54.7 \pm 10.8 , range $31-75$ years) did not differ significantly from that of the HBV cirrhotic patients group (51.7 \pm 9.6 ; range $31-75$ years) $(\mathrm{P}=0.069)$. The mean BMI of the control group (24.54 \pm 3.14 , range $18.3-34.2 \mathrm{~kg} / \mathrm{m} 2)$ was not significantly different from that of the HBV cirrhotic patient group (23.88 \pm 4.20 , range $16.7-34.1 \mathrm{~kg} / \mathrm{m} 2)(\mathrm{P}=0.224) .25 \%(20 / 80)$ of the patients with hepatitis B cirrhosis and 20\% (16/80) in the healthy control group smoked. In the HBV cirrhosis group, $75 \%(27 / 36)$ and $62.5 \%(25 / 40)$ women in the control group had menopause. 
Table 1 Characteristics of patients

\begin{tabular}{llll}
\hline Variables & $\begin{array}{l}\text { HBV cirrhosis } \\
(\mathrm{n}=80)\end{array}$ & $\begin{array}{l}\text { Controls } \\
(\mathrm{n}=80)\end{array}$ & P-value \\
\hline Age $($ years, mean $\pm \mathrm{SD})$ & $51.7 \pm 9.6$ & $54.7 \pm 10.8$ & 0.069 \\
Male, $\mathrm{n}(\%)$ & $44(55.00)$ & $40(50.00)$ & 0.527 \\
BMI $\left(\mathrm{kg} / \mathrm{m}^{2}\right.$, mean $\left.\pm \mathrm{SD}\right)$ & $23.88 \pm 4.20$ & $24.54 \pm 3.14$ & 0.224 \\
Smoke, $\mathrm{n}(\%)$ & $20(25.00)$ & $16(20.00)$ & 0.449 \\
Menopause, $\mathrm{n}(\%)$ & $27(75.00)$ & $25(62.5)$ & 0.242 \\
\hline
\end{tabular}

Herein, we found that $70 \%(56 / 80)$ of the HBV cirrhotic patients had decreased $\mathrm{BMD}$ as compared to $35 \%(28 / 80)$ of the control group. Compared to the control group, the HBV cirrhotic patients had significantly higher rates of osteoporosis $(27.5 \%$ vs. $7.5 \%$, $\mathrm{P}<0.001)$. In the HBV cirrhotic group, 5 patients experienced fragility fractures; one of these patients suffered right wrist and left ankle fractures, respectively, while no fractures occurred in the control group. The BMD values of the lumbar spine, femoral neck, tro- chanter, and total hip in the HBV cirrhotic patients and controls are shown in Table 2. The BMD values in all scanned regions were significantly lower in the HBV cirrhotic patients as compared to the controls. Furthermore, the BMD was lower in the patients in the $\mathrm{C}$ grade subgroup as compared to the $\mathrm{A}$ and $\mathrm{B}$ grade subgroups, and the lumbar spine BMD was significantly lower in the $\mathrm{C}$ grade subgroup than that in the $\mathrm{A}$ and $\mathrm{B}$ grade subgroups. The femoral neck, trochanter, and total hip BMD were also lower in the Child-Pugh C subgroup, albeit not significantly (Table 3).

Table 2 Bone mineral density in the HBV group and the control group

\begin{tabular}{llll}
\hline Site & $\begin{array}{l}\mathrm{BMD}\left(\mathrm{g} / \mathrm{cm}^{2}\right) \\
\text { HBV cirrhosis } \\
(\mathrm{n}=80)\end{array}$ & $\begin{array}{l}\mathrm{BMD}\left(\mathrm{g} / \mathrm{cm}^{2}\right) \\
\text { Controls } \\
(\mathrm{n}=80)\end{array}$ & P-value \\
\hline LS & $0.90 \pm 0.15$ & $1.00 \pm 0.14$ & \\
FN & $0.72 \pm 0.12$ & $0.76 \pm 0.11$ & $<0.001$ \\
WT & $0.65 \pm 0.10$ & $0.69 \pm 0.09$ & 0.029 \\
Tro & $1.08 \pm 0.16$ & $1.13 \pm 0.14$ & 0.006 \\
TH & $0.89 \pm 0.13$ & $0.93 \pm 0.11$ & 0.040 \\
\hline
\end{tabular}

LS: lumbar spine; FN: femoral neck; Tro: trochanter; TH: total hip 
Table 3 Association between BMD and Child-Pugh grade of HBV cirrhotic patients

\begin{tabular}{|c|c|c|c|c|c|c|}
\hline \multirow{2}{*}{$\begin{array}{l}\text { Site } \\
(\mathrm{g} / \mathrm{cm}\end{array}$} & \multirow{2}{*}{$\begin{array}{l}\mathrm{BMD}\left(\mathrm{g} / \mathrm{cm}^{2}\right) \\
\text { Child-Pugh } \\
(\mathrm{n}=57)\end{array}$} & \multirow{2}{*}{\multicolumn{2}{|c|}{$\begin{array}{l}\mathrm{BMD}\left(\mathrm{g} / \mathrm{cm}^{2}\right) \\
\text { A Child-Pugh } \\
(\mathrm{n}=16) \\
\end{array}$}} & \multirow[b]{2}{*}{ B } & \multirow{2}{*}{$\begin{array}{l}\operatorname{BMD}\left(\mathrm{g} / \mathrm{cm}^{2}\right) \\
\text { Child-Pugh C }(\mathrm{n}=7)\end{array}$} & \multirow[b]{2}{*}{ P-value } \\
\hline & & & & & & \\
\hline LS & $0.90 \pm 0.14$ & & $0.93 \pm 0.14$ & & $0.77 \pm 0.19$ & 0.039 \\
\hline FN & $0.73 \pm 0.12$ & & $0.72 \pm 0.12$ & & $0.68 \pm 0.11$ & 0.567 \\
\hline WT & $0.66 \pm 0.11$ & & $0.66 \pm 0.12$ & & $0.59 \pm 0.07$ & 0.296 \\
\hline Tro & $1.08 \pm 0.16$ & & $1.08 \pm 0.19$ & & $1.04 \pm 0.17$ & 0.763 \\
\hline $\mathrm{TH}$ & $0.89 \pm 0.13$ & & $0.90 \pm 0.15$ & & $0.81 \pm 0.13$ & 0.269 \\
\hline
\end{tabular}

LS: lumbar spine; FN: femoral neck; Tro: trochanter; TH: total hip

Serum $\beta$-CTX was significantly higher in the ChildPugh $\mathrm{C}$ grade subgroup than in the $\mathrm{A}$ and $\mathrm{B}$ grade subgroups, and $25(\mathrm{OH}) \mathrm{D} 3$ was significantly lower in the Child-Pugh $\mathrm{C}$ grade subgroup than in the $\mathrm{A}$ and $\mathrm{B}$ grade subgroups. The levels of P1NP and OC were higher in the Child-Pugh $\mathrm{C}$ grade subgroup, but the difference was not statistically significant (Table 4).
Multivariate logistic regression analysis was performed in the presence of osteoporosis as the dependent variable and gender, age, BMI, smoking, and hepatitis B as independent variables. The results showed that age and hepatitis B were risk factors for osteoporosis. Interestingly, the risk of osteoporosis in patients with chronic hepatitis B was higher than that in healthy controls ( $\mathrm{Ta}-$ ble 5).

Table 4 Association between bone markers and Child-Pugh scores in HBV cirrhotic patients

\begin{tabular}{|c|c|c|c|c|}
\hline $\begin{array}{l}\text { Variable } \\
(\mathrm{ng} / \mathrm{mL})\end{array}$ & $\begin{array}{l}\text { Child-Pugh A } \\
(\mathrm{n}=57)\end{array}$ & $\begin{array}{l}\text { Child-Pugh B } \\
(\mathrm{n}=16)\end{array}$ & $\begin{array}{l}\text { Child-Pugh C } \\
(\mathrm{n}=7)\end{array}$ & P-value \\
\hline P1NP & $60.53 \pm 42.01$ & $47.96 \pm 23.31$ & $76.28 \pm 17.98$ & 0.236 \\
\hline $\mathrm{OC}$ & $18.19 \pm 9.69$ & $16.64 \pm 10.76$ & $18.59 \pm 9.15$ & 0.841 \\
\hline$\beta$-СТX & $0.47 \pm 0.28$ & $0.57 \pm 0.40$ & $1.01 \pm 0.59$ & 0.001 \\
\hline $25(\mathrm{OH}) \mathrm{D} 25(\mathrm{OH}) \mathrm{D} 3$ & $21.00 \pm 10.42$ & $15.73 \pm 7.35$ & $12.38 \pm 8.62$ & 0.029 \\
\hline
\end{tabular}

P1NP: procollagen type 1 amino-terminal propeptide; OC: serum osteocalcin; $\beta$-CTX: $\beta$-Cterminal telopeptides of type-I collagen; 25(OH)D3: 25-Hydroxyvitamin D3 
Table 5 Risk factors of osteoporosis in patients with chronic hepatitis B

\begin{tabular}{lllll}
\hline & & & \multicolumn{2}{c}{$95 \%$ CI for OR } \\
\cline { 4 - 5 } Effect & P-value & OR & Lower & Upper \\
\hline Gender & 0.004 & 0.191 & 0.061 & 0.599 \\
Age & 0.003 & 1.056 & 1.019 & 1.094 \\
BMI & 0.025 & 0.895 & 0.813 & 0.986 \\
Smoking & 0.133 & 1.815 & 0.834 & 3.950 \\
HBV & 0.000 & 5.299 & 2.566 & 10.943 \\
\hline
\end{tabular}

\section{Discussion}

The current study demonstrated that the prevalence of osteoporosis (27.5\%) was higher in CHB patients with cirrhosis as compared to the controls. The progression of cirrhosis from A to C grades of the Child-Pugh classification was associated with a decreased BMD, which was consistent with the literature ${ }^{11}$. The lumbar spine BMD was significantly lower in the Child-Pugh C grade subgroup than that in the $\mathrm{A}$ and $\mathrm{B}$ grade subgroups in the cirrhotic patients. In addition, $\beta$-CTX was elevated with the progression of cirrhosis.

Recently, a large-scale population-based cohort study from Taiwan demonstrated that chronic HBV infection increases the risk of osteoporosis ${ }^{12}$. Interestingly, the risk remained higher in the cohort, even after adjusting for age, sex, and complications of cirrhosis, chronic renal insufficiency, thyroid diseases, diabetes, and hypertension $^{12}$. In the current study, 5/80 patients had experienced fragility fractures in the cirrhotic group, while no patient had a fracture in the control group. The association between HBV infection and osteoporotic fractures was statistically significant. However, Chen et al. showed that the incidence of osteoporotic fractures was not statistically higher in patients with $\mathrm{HBV}$ as compared to those without HBV infection ${ }^{12}$. Intriguingly, Byrne et al. found that chronic HBV infection increased the risk of hip fracture in the patients of all races except for Asians ${ }^{13}$. The differences between these studies highlighted why the patients enrolled in our study were all cirrhotic patients. HBV infection was a less important risk factor for osteoporosis as compared to cirrhosis. Therefore, a large-scale study is essential to further explore the correlation between HBV-related cirrhosis and osteoporotic fractures.
In the present study, $\beta$-CTX was significantly higher in cirrhotic patients of Child-Pugh grade C. We hypothesized that severe cirrhotic patients exhibit accelerated bone resorption. The lumbar spine BMD decreased with the progression of cirrhosis. The mechanism of association between the decreased BMD and severity of cirrhosis has not yet been fully elucidated. The production of inflammatory cytokines, such as interleukin-6 (IL-6), tumor necrosis factor-alpha (TNF- $\alpha$ ), and IL-1 increases the receptor activator of the nuclear factor kappa-B ligand, which further promotesosteoclastogenesis and bone resorption, resulting in a decrease in $\mathrm{BMD}^{14-17}$. Hepatic decompensation or cirrhosis also prevents bone formation by impairing the production of $25(\mathrm{OH}) \mathrm{D} 3$ and insulin-like growth factor- ${ }^{18,19}$. Moreover, hypogonadism with decreased levels of estrogen and testosterone accelerates the bone loss, primarily due to the increased osteoclast activity, which is often observed in the decompensated livers ${ }^{20}$. Furthermore, a decompensated liver impairs the collagen-binding of the bone matrix and inhibits osteoblast function ${ }^{21}$. Finally, increased bilirubin inhibits osteoblast proliferation ${ }^{22-24}$. Also, we provided data on the changes in 25(OH)D3 and $\beta$-CTX and their clinical significance in $\mathrm{CHB}$ patients with cirrhosis. A remarkably reduced level of $25(\mathrm{OH}) \mathrm{D} 3$ was observed in cases of severe cirrhosis. Similar results have been reported in a previous study of cirrhotic patients ${ }^{25}$. The possible underlying mechanisms include decreased hepatic hydroxylation of vitamin D, intestinal malabsorption, lessened sunlight exposure, and reduced dietary intake. Vitamin $\mathrm{D}$ deficiency decreases calcium resorption and activates bone resorption to maintain the level of calcium in the blood, resulting in bone loss ${ }^{26}$. In addition, impaired hydroxylation of vitamin D3 to 25-OH-D3 in the liver 
promotes bone loss and decreased bone formation ${ }^{27}$. The increased osteoclast function and bone resorption partially explained the high rates of low BMD in cirrhosis. Regression analysis showed that age and hepatitis B were risk factors for osteoporosis. Therefore, the diagnosis and treatment of osteoporosis in $\mathrm{CHB}$ patients should be investigated to prevent osteoporosis and retain the quality of life of the patients.

The major strength of the current study was that bone turnover markers were evaluated as potential mechanisms of bone disease in HBV-related cirrhotic patients. A major limitation of this study was the cross-sectional design, although the changes in BMD were estimated longitudinally. Also, whether the results could be extrapolated to all the cirrhotic patients owing to the small sample size of the Child-Pugh grade $\mathrm{C}$ cirrhotic patients was unclear. Furthermore, the controls did not present results for the bone turnover markers.

\section{Conclusion}

Osteoporosis was highly prevalent among $\mathrm{CHB}$ patients with cirrhosis. Child-Pugh grade $\mathrm{C}$ cirrhotic patients showed enhanced osteoclastic activity, indicated by increased $\beta$-CTX. Enhanced bone resorption accounted for the increased risk of osteoporosis in patients with severe cirrhosis. Thus, osteoporosis in HBV-related cirrhotic patients should be under intensive focus. $\mathrm{CHB}$ patients diagnosed first time with cirrhosis should undergo DXA examination for the early diagnosis of the onset of osteoporosis. Therefore, patients with cirrhosis are recommended to undergo BMD assessments annually. After osteoporosis is diagnosed in cirrhotic patients, drug therapy that inhibit the activity of osteoclasts must be prescribed to reduce the risk of fractures.

\section{Competing interests}

The authors have declared that no competing interests exist.

\section{Acknowledgments}

None.

\section{Funding}

Construction of high-level health technical personnel in the Beijing health system (2014-3-093). China's National Science and Technology Major Project (2018ZX10715005-003-002).

\section{References}

1. Global, regional, and national life expectancy, all- cause mortality, and cause-specific mortality for 249 causes of death, 1980-2015: a systematic analysis for the Global Burden of Disease Study 2015. Lancet. 2016;388 (10053):1459-1544.

2. Collier J. Bone disorders in chronic liver disease. Hepatology. 2007;46 (4):1271-1278.

3. Collier JD, Ninkovic M, Compston JE. Guidelines on the management of osteoporosis associated with chronic liver disease. Gut. 2002;50 Suppl 1:11-9.

4. Liao CY, Chung CH, Chu P, Wei KY, Feng TM, Lin $\mathrm{FH}$, et al. Increased risk of osteoporosis in patients with primary biliary cirrhosis. PLoS One. 2018;13 (3): $\mathrm{e} 0194418$.

5. Tang R, Wei Y, Li Z, Chen H, Miao Q, Bian Z, et al. A Common Variant in CLDN14 is Associated with Primary Biliary Cirrhosis and Bone Mineral Density. Sci Rep. 2016;6:19877.

6. Otete H, Deleuran T, Fleming KM, Card T, Aithal GP, Jepsen P, et al. Hip fracture risk in patients with alcoholic cirrhosis: A population-based study using English and Danish data. J Hepatol. 2018;69 (3):697-704.

7. Terrault NA, Bzowej NH, Chang KM, Hwang JP, Jonas MM, Murad MH. AASLD guidelines for treatment of chronic hepatitis B. Hepatology. 2016;63 (1):261283.

8. Assessment of fracture risk and its application to screening for postmenopausal osteoporosis. Report of a WHO Study Group. World Health Organ Tech Rep Ser. 1994;843:1-129.

9. Vasikaran S, Eastell R, Bruyere O, Foldes AJ, Garnero P, Griesmacher A, et al. Markers of bone turnover for the prediction of fracture risk and monitoring of osteoporosis treatment: a need for international reference standards. Osteoporos Int. 2011;22 (2):391-420.

10. Li J, Zhang H, Yang C, Li Y, Dai Z. An overview of osteocalcin progress. J Bone Miner Metab. 2016;34 (4):367-379.

11. Hajiabbasi A, Shafaghi A, Fayazi HS, Shenavar Masooleh I, Hedayati Emami MH, Ghavidel Parsa P, et al. The factors affecting bone density in cirrhosis. Hepat Mon. 2015;15 (4):e26871.

12. Chen $\mathrm{CH}$, Lin CL, Kao CH. Association Between Chronic Hepatitis B Virus Infection and Risk of Osteoporosis: A Nationwide Population-Based Study. Medicine (Baltimore). 2015;94 (50):e2276.

13. Byrne DD, Newcomb CW, Carbonari DM, Nezamzadeh MS, Leidl KB, Herlim M, et al. Risk of hip fracture associated with untreated and treated chronic hepatitis B virus infection. J Hepatol. 2014;61 (2):210-218. 14. Gilbert L, He X, Farmer P, Rubin J, Drissi H, van Wijnen AJ, et al. Expression of the osteoblast differen- 
tiation factor RUNX2 (Cbfa1/AML3/Pebp2alpha A) is inhibited by tumor necrosis factor-alpha. J Biol Chem . 2002;277 (4):2695-2701.

15. Gonzalez-Calvin JL, Gallego-Rojo F, Fernandez-Perez R, Casado-Caballero F, Ruiz-Escolano E, Olivares EG. Osteoporosis, mineral metabolism, and serum soluble tumor necrosis factor receptor $\mathrm{p} 55$ in viral cirrhosis. J Clin Endocrinol Metab. 2004;89 (9):4325-4330. 16. McGregor NE, Murat M, Elango J, Poulton IJ, Walker EC, Crimeen-Irwin B, et al. IL-6 exhibits both cis- and trans-signaling in osteocytes and osteoblasts, but only trans-signaling promotes bone formation and osteoclastogenesis. J Biol Chem. 2019;294 (19):78507863.

17. Jules J, Feng X. In vitro investigation of the roles of the proinflammatory cytokines tumor necrosis factor-alpha and interleukin-1 in murine osteoclastogenesis. Methods Mol Biol. 2014;1155:109-123 PubMed .

18. Gallego-Rojo FJ, Gonzalez-Calvin JL, Munoz-Torres M, Mundi JL, Fernandez-Perez R, Rodrigo-Moreno D. Bone mineral density, serum insulin-like growth factor I, and bone turnover markers in viral cirrhosis. Hepatology. 1998;28 (3):695 PubMed -699.

19. Corazza GR, Trevisani F, Di Stefano M, De Notariis S, Veneto G, Cecchetti L, et al. Early increase of bone resorption in patients with liver cirrhosis secondary to viral hepatitis. Dig Dis Sci. 2000;45 (7):1392 PubMed -1399 .

20. George J, Ganesh HK, Acharya S, Bandgar TR, Shivane V, Karvat A, et al. Bone mineral density and disorders of mineral metabolism in chronic liver disease. World J Gastroenterol. 2009;15 (28):3516-3522.

21. Nakchbandi IA. Osteoporosis and fractures in liver disease: relevance, pathogenesis and therapeutic implications. World J Gastroenterol. 2014;20 (28):9427-9438. 22. Samra NM, Emad El Abrak S, El Dash HH, El Said El Raziky M, El Sheikh MA. Evaluation of vitamin D status bone mineral density and dental health in children with cholestasis. Clin Res Hepatol Gastroenterol. 2018;42 (4):368-377.

23. Ruiz-Gaspa S, Dubreuil M, Guanabens N, Combalia A, Peris P, Monegal A, et al. Ursodeoxycholic acid decreases bilirubin-induced osteoblast apoptosis. Eur J Clin Invest. 2014;44 (12):1206 PubMed -1214.

24. Ruiz-Gaspa S, Martinez-Ferrer A, Guanabens N, Dubreuil M, Peris P, Enjuanes A, et al. Effects of bilirubin and sera from jaundiced patients on osteoblasts: contribution to the development of osteoporosis in liver diseases. Hepatology. 2011;54 (6):2104 PubMed -2113. 25. Lai JC, Bikle DD, Lizaola B, Hayssen H, Terrault NA, Schwartz JB. Total $25(\mathrm{OH})$ vitamin D, free $25(\mathrm{OH})$ vitamin $\mathrm{D}$ and markers of bone turnover in cirrhotics with and without synthetic dysfunction. Liver Int. 2015;35 (10):2294 PubMed -2300.

26. Holick MF. Vitamin D deficiency. $N$ Engl J Med. 2007;357 (3):266-281.

27. van Leeuwen JP, van Driel M, van den Bemd GJ, Pols HA. Vitamin D control of osteoblast function and bone extracellular matrix mineralization. Crit Rev Eukaryot Gene Expr. 2001;11 (1-3):199-226. 